\title{
UTILISATION DU MAİS GRAIN HUMIDE CONSERVÉ A L'ACIDE PROPIONIQUE POUR L'ENGRAISSEMENT DES TAURILLONS
}

\author{
Y. GEAY, C. MALTIRRE et P. THIVEND* \\ avec la collaboration technique de Paulette Joursatx*, \\ Bernadette Iassalas*, Robert Jallizk, G. Cuvlle, P. IÉxalli** \\ Laboratoire de la Production de Viande, \\ * Station de Rechrches sur l'Élevage des Ruminants, \\ Contre do Recherch's de Clermont-Ferrand, I. N.R. A., \\ Theix, Saint Genès Champanelle, 63110 Beaumont \\ ** Domaine de Bressonvilliers, I. N. R.A.
}

\section{RÉSUMÉ:}

Deux expériences d'engraissement de taurillons ont été effectuées pour étudier l'influence du traitement à l'acide propionique du mä̈s grain humide sur sa valeur nutritive. Dans chaque cas, le lot expérimental recevant le maïs humicle traité puis aplati, a été comparé à un lot témoin recevant le mails sec broyé et aggloméré. Le grain a représenté jo p. Ioo de la matière sèche de la ration lors de la $I^{r e}$ expérience ct 7 o p. 1 oo lors de la seconde, la luzerne déshydratée condenséc constituant le reste de la ration.

Soixante taurillons ont été engraissés lors de ces 2 expériences durant respectivement 139 jours pour la I $^{\mathrm{rc}}$ et $9^{6}$ jours pour la seconde. La première expérience a comporté 3 lots d'animaux ; 2 d'entre eux ont reçu une quantité égale de matière sèche de grain, soit sous forme humicle, soit sous forme sèche, et le troisième une ration de grain humide à volonté. La seconde expérience a comporté z lots recevant soit le maïs sec, soit le maïs humide aplati.

L'acide propionique a permis de conserver le grain humide (68 et $6+, 5$ p. Ioo de matière sèclie) durant une période de 9 mois, avec une très faible proportion de pertes.

Nous n'avons pas noté au cours de ces 2 expériences une influence significative du traitement sur le gain de poids vif, le poids et la composition des carcasses, mais une légère diminution des quantités ingérées et par suite une légère amélioration de l'efficacité alimentaire ( 1,8 et 3,3 p. 10o) des rations.

Le traitement à l'acicle propionique a entraîné une diminution notable de la digestibilité les principaux constituants de la ration. Si la quantité d'acides gras volatils formés au niveau du rumen a été identique, la composition du mélange d'acides gras volatils a été modifiée dans un sens favorable à une meilleure utilisation de l'énergie (augmentation de la quantité d'acide butyrique, diminution de l'acide acétique) lorsque les animaux ont reçu le maïs humide.

Si ces résultats ne mettent pas en évidence des différences significatives, ils traduisent cependaut, dans chaque expérience, une légère amélioration de la valeur nutritive du maïs lorsqu'il est conservé humide avec de l'acide propionique. 


\section{INTRODUC'TION}

Lorsque le maïs destiné à l'alimentation animale est récolté sous forme de grains entiers, il est en règle générale stocké après un séchage amenant le taux de matière sèche à $85 \mathrm{p}$. Ioo. Ce procédé nécessite un équipement coûteux dont la capacité est limitée et ne permet pas d'absorber rapidement une récolte abondante. Ein outre, lorsque le grain est en proportion importante dans la ration, il est préférable qu'il soit condensé (broyé et aggloméré) si l'on veut régulariser l'ingestion des animaux et limiter les accidents digestifs consécutifs à la consommation de farine ; cela accroît le coût de l'alimentation.

Des traitements technologiques nombreux et complexes ont été étudiés dans le but d'améliorer la valeur alimentaire du grain sec (HANke et JordAN, I963; FoxTENot et Hopkins, I965; BÉranger, ThIVEnd et JARrigE, I972). Mais l'amélioration enregistrée a généralement été faible et insuffisante pour justifier le coût de ces traitements dans les conditions actuelles françaises.

Aussi, les méthodes qui permettent de stocker et d'utiliser directement le maïs humide, peuvent-elles présenter un certain intérêt : elles évitent le séchage et la fabrication de granulés, et permettent dans certaines conditions d'anléliorer l'appétibilité et la valeur nutritive du grain humide par rapport au grain sec (BEESor et Perry, I958; Zogg et al., ig6r; MacCariree et Merrili, ig66; MacGinty et RigGs, I967; WHITE et al., Ig69).

Une de ces méthodes de conservation consiste à pulvériser de l'acide propionique sur le grain récolté humide. Ce procédé a été utilisé avec succès pour conserver l'orge (B. P. Chemicals, r968) ou le mais destiné à l'alimentation des pores (JoNes, DONEFEr et Eillotot, I970 ; FÉvrier, Bourdon et Chambolle, I972), des agneaux (Thériez, LE Du et Moléxat, I973), des vaches laitières et des génisses (Jones, DONEFER et EliLIOT, I970), et des bouvillons à l'engrais (Forsyth, Mowat et Stone, I972). Ce procédé apparaissant a priori simple et peu onéreux, nous en avons étudié l'application à la conservation du maïs récolté humide destiné à l'alinentation des taurillons. Au cours de 2 expériences successives, nous avons comparé les performances d'engraissement de ces animaux recevant, soit le maîs sec condensé (broyé et aggloméré), soit le maïs humide aplati; le grain représentant jo p. Ioo de la matière sèche de la ration lors de la $\mathrm{I}^{\mathrm{r}} \mathrm{e}$ expérience et $70 \mathrm{p}$. Ioo lors de la seconde (1).

\section{MA'TÉRIEI, ETT MÉTHODES}

\section{Expérienci I}

\section{Principe.}

Nous avons comparé, au cours de cette expéricnce réalisée au C . K.Z. . de Theix, trois régimes comportant $50 \mathrm{p}$. Ioo de la matière sèche sous forme de luzerne déshydratée condensée et $50 \mathrm{p}$. I 00 sous forme de maïs grain distribué soit sec et condensé pour l'un cles régimes, soit humide et aplati

(1) Les résultats de ces expériences ont fait l'objet de comptes rendus dans le Bulletin Technique du C.R.Z.V. de Theix (Glay et Lí́nard, ig7i ; Malterrli et Giay', I973). Lreong et Moal (I973) en ont fait état au cours du Simposiun Interuational sur la conservation des praius récoltés humides. 
pour les 2 autres. Le régime comportant le maïs sec et l'un des régimes comportant le maïs humide ont été distribués à volonté (" maïs sec net "maïs humide ad libitum"). Le second régime comportant le maïs humide a été distribué en quantité de matière sèche égale à celle consommée par les animaux recevant le régime " maïs sec".

\section{Traitement du maïs.}

Pour réaliser cette expérience, to tonnes de maïs grain INRA 258 récoltées à 68 p. Ioo de matière sèche, ont été arrosées d'acide propionique au taux de I,5 p. Ioo. L'acide provenait d'un pulvérisateur placé à l'entrée de la vis d'Archimède utilisée pour véhiculer le grain vers le lieu de stockage. Ces 40 tonnes ont été entreposées en vrac, à l'intérieur d'un bâtiment couvert. Une bâche plastique entourait et recouvrait le tas pour protéger le grain de la poussière et faciliter les mesures de température par sondage. Le maïs, ainsi conservé humide, a été aplati entre 2 rouleaux, chaque jour, avant d'être distribué aux animaux. Par ailleurs, 37,5 tonnes du même maîs ont été séchées jusqu'au taux de $85 \mathrm{p}$. Ioo de matière sèche, puis broyées à la grille de $3 \mathrm{~mm}$ et agglomérées à la filière de ro $\mathrm{mm}$.

Au cours de sa conservation, le grain humide a fait l'objet des mesures suivantes : relevé des températures à différents endroits du silo tous les 3 jours, prélèvement d'échantillons représentatifs (au début, au milieu et en fin de conservation) sur lequel on a mesuré les teneurs en matière sèche et en amidon (Thivend, Mercier, Guilbot, I972).

\section{Animaux et alimentation.}

A partir de zi taurillons de race Salers et zi taurillons croisés Rouge des Filandres $\times$ Salers, I 4 blocs de 3 animaux ont été constitués, les animaux de chaque bloc étant aussi semblables que possible, sur la base du poids et de l'âge. Dans chacun des blocs, les animaux ont reçu respectivement l'un des trois régimes précédents; les $\mathrm{I}_{4}$ taurillons de chaque régime ayant été répartis de la façon suivante : 6 en stabulation libre ( 3 Salers et 3 Croisés) et 8 en stabulation entravée (4 Salers et + Croisés).

Pour limiter le tri des aliments par les animaux, le maïs et la luzerne ont été mélangés dans l'auge au moment de la distribution. Chaque jour, on a distribué en plus à tous les animaux roo g de complément minéral que l'on a mélangé au maïs. Tous les animaux ont pu consommer la paille de leur litière.

\section{Mesuves.}

\section{- Quantités ingévées et croissance.}

Chaque semaine, on a mesuré les quantités d’aliments ingérées pendant 6 jours par chaque animal entravé et par les lots d'animaux en stabulation libre. Les animaux ont été pesés pendant 2 jours consécutifs, à la même heure, au moment de la mise en lots, en début de période expérimentale, et avant de partir pour l'abattoir, ainsi que toutes les 4 semaines durant la période expérimentale.

\section{- Utilisation digestive.}

Nous avons mesuré la digestibilité des deux rations distribuées à volonté sur deux taurillons

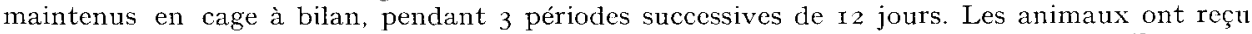
la même proportion de paille que celle consommée par les animaux en lots (io p. Ioo). En outre, la digestion de ces deux rations a été étudiée sur 2 génisses munies d'une canule réentrante du duodénum et sur 2 bouvillons munis d'une canule du rumen. Cependant, la proportion de paille ingérée (25 p. IOO) a été plus importante que dans la ration des animaux en digestibilité. Pour chaque régime, nous avons mesuré au cours d'une période de 8 heures l'évolution postprandiale de la composition du mélange d'acides gras volatils du jus de rumen. Au niveau du duodénum, nous avons recueilli pendant une période de 12 heures (correspondant à l'intervalle entre les deux repas) la totalité du contenu digestif. Chaque heure, une partie aliquote était prélevée, le reste du contenu étant réintroduit à l'aide d'une pompe. Sur la moyenne de I 2 échantillons ainsi obtenus, mélangés et lyophilisés, nous avons déterminé la teneur en matière sèche, matière organique, matières azotées et amidon. Ces mesures ont été répétées 4 fois à des intervalles d'au moins $4^{8}$ heures pendant 2 semaines consécutives.

\section{- Abattage et composition des carcasses.}

Les animaux ont été abattus par bloc de 3 (un animal de chaque lot ayant le même mode de. stabulation) lorsque l'état d'engraissement du taurillon du lot 3 a été jugé satisfaisant, en moyenne au bout de I $39 \neq 30$ jours. 
A l'abattage, on a mesuré le poids des carcasses, le rendement en carcasse ct le poids du " $5^{\mathrm{e}}$ quartier". La carcasse de chaque animal a été notée au point de vue de sa conformation et de son état d'engraissement et a fait l'objet d'un certain nombre de mensurations et de pesées après découpe : mesure de la longueur de la carcasse et de la cuisse (distance jarret-symphyse), épaisseur de la cuisse et du faux-filet (VISSAC, I959), poids du "pan traité " (DUmont, I956), ainsi que de l'ensemble épaule +collier.

La composition des carcasses a été estimée à partir de celle de la " I I côte ", grâce aux relations établies par GEAY et BÉraNGer (I969).

\section{Expérience $I I$}

\section{Principe.}

Au cours de cette expérience, les animaux ont reçu à volonté une ration à base de maìs grain, distribué soit sous forme sèche et condensée (régime maîs sec), soit sous forme humide, conservé à l'acide propionique et aplati (régime maïs humide). L'ensemble des animaux a reçu en supplément du maïs grain une quantité limitée de luzerne déshydratée compactée (presse à filières de $\mathbf{2} 2 \mathrm{~mm}$ de diamètre), de telle sorte que cette dernière représente $30 \mathrm{p}$. I oo de la matière sèche de la ration. La luzerne était distribuée séparément avant la ration de maïs et l'ajustement était réalisé chaque semaine sur la base des quantités de maïs ingérées la semaine précédente. Cent vingt grammes du complément minéral utilisé lors de la I re expérience, ont été distribués chaque jour à chaque animal Tous les taurillons ont pu consommer la paille de leur litière, mais la quantité ingérée n'a pas été estimée.

\section{Dispositif expévimental.}

Pour réaliser cette expérience, I6,5 tonncs de maïs grain $I N R A 258$, récoltées à une teneur en matière sèche de $64,5 \mathrm{p}$. IoO, ont également été traitées à l'acide propionique au taux de I, 5 p. Ioo. Ce maïs a été entreposé de la même façon que précédemment. Il n'a cependant pu faire l'objet de contrôles de température; toutefois, les quantités de grains moisis ont été mesurées. Par ailleurs, I 3,5 tonnes du même maïs ont été séchées jusqu'au taux de $85 \mathrm{p}$. Ioo de matière sèche, broyées et agglomérées. Trente-cinq jours après le traitement du grain humide, 2 lots de I 3 taurillons Frisons Danois, de poids et d'àge semblables, ont été constitués et placés en stabulation libre. Chaque lot a été réparti en 2 groupes d'animaux, l'un comprenant les 7 plus lourds, l'autre les 6 plus légers. L'ensemble des animaux a été abattu à deux dates différentes : les I 4 taurillons lourds après 79 jours d'engraissement et les $\mathrm{I} 2$ taurillons légers après I $2 \mathrm{I}$ jours. Lees mesures réalisées à l'abattage ont été les mêmes que dans l'expérience précédente.

\section{RÉSULTATS}

\section{Conservation}

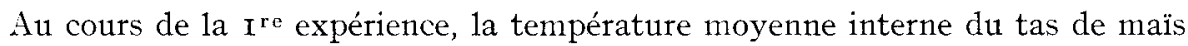
humide, après s'être élevée de $5^{\circ}$ à $6^{\circ} \mathrm{C}$ durant les 5 premiers jours, s'est abaissée régulièrement et a suivi l'évolution de la température extérieure. Divers nodules de grains moisis ayant apparemment échappé au traitement, ont été retirés du tas lors de la distribution du mais aux animaux. Les grains apparemment sains qui entouraient certains nodules particulièrement volumineux ont été traités à nouveau à l'acide propionique au taux de $0,5 \mathrm{p}$. Ioo. Le poids total des pertes a représenté $4 \mathrm{p}$. Ioo de la quantité stockée, proportion beaucoup plus importante que dans le second essai où nous n'avons constaté que $\mathrm{I}, 2 \mathrm{p}$. Ioo de grains moisis.

Durant 160 jours, la teneur en matière sèche du grain est restée stable : 68,6 $土$ 0,8 p. I00, puis elle s'est élevée pour atteinđre 72 p. 100, 270 jours après le traitement. En revanche, la teneur en amidon est restée constante tout au long des 270 jours de conservation : $69,9 \pm \mathrm{I}, \mathrm{I}$ p. Ioo de la matière sèche. Enfin, le pouvoir germinatif du grain traité a été nul. 


\section{Etat sanitaire des animanx}

Durant le $I^{\text {er }}$ essai, deux animaux en stabulation libre, recevant la ration à base de maïs sec, ont météorisé fréquemment. L'un des animaux (de race Salers) a dût être abattu, l'autre (Ronge des Flandres $\times$ Salers) a été retiré du lot et mis en stabulation entravée où il a reçu, à volonté, la ration comportant le maïs humide aplati sans présenter de troubles digestifs. Lors du second essai, un animal du régime comportant le maîs humide, malade peu de jours après le début de l'expérience, a dû être éliminé. Dans les 2 essais, les résultats provenant des blocs qui comportaient des animaux malades n'ont pas été utilisés.

\section{('roissance et quantités ingérées (tabl. I et 2)}

Les gains de poids vif des animaux ont été élevés: respectivement I 520 , I 505 et I $587 \mathrm{~g} / \mathrm{j}$ pour les régimes "maïs sec ", " maïs humide limité " et " maïs humide ad libitum" du I ${ }^{\mathrm{e}}$ essai ; I 526 et $\mathrm{I} 507 \mathrm{~g} / \mathrm{j}$ respectivement pour les régimes " maïs sec " et " maïs humide " du $2^{\mathrm{e}}$ essai (tabl. I et 2 ). Mais aucune différence significative n'est apparue entre les régimes.

Le maïs grain humide conservé à l'acide propionique et distribué à rolonté a été consommé en quantités égales ( $\mathrm{I}^{\mathrm{r}}$ expérience) ou légèrement inférieures au maïs $\sec \left(3 \mathrm{p}\right.$. Ioo, $2^{\mathbf{e}}$ expérience) (tabl. I et 2 ).

Dans l'ensemble, les quantités de matière sèche ingérées par kg de gain de poids vif ont été très voisines sauf lors du premier essai où les animaux recevant le maïs humide à volonté ont ingéré en moins 3,3 p. Ioo de matière sèche par $\mathrm{kg}$ de gain.

Signalons enfin qu'indépendamment de la nature de la ration, le gain de poids vif des taurillons Croisés Rouge des Flandres $\times$ Salers a été significativement supérieur $(\mathrm{P}<0,05)$ de $24,6 \mathrm{p}$. Ioo à celui des Salers ( 705 contre $\mathrm{I} 368 \mathrm{~g} / \mathrm{j}$ ). Le gain de poids vif des taurillons Frisons Danois, les plus lourds au départ a été supérieur de 6,8 p. Ioo à celui des animaux pluss légers ( $\mathrm{I} 55^{8}$ contre I $45^{8} \mathrm{~g} / \mathrm{j}$ ), mais la différence n'a pas été significative.

\section{Utilisation digestive}

La digestibilité de la ration à base de maïs sec (matière sèche, matière organique, matières azotées et amidon) a été plus élevée que celle de la ration à base de maîs humide (tabl. 3). L'importance de la digestion de la matière sèche, de la matière organique et de l'amidon, dans les réservoirs gastriques, a été identique pour les deux types de régimes (tabl. 3). En conséquence, la digestion intestinale de ces aliments a été plus importante avec le maìs sec qu'avec le maïs humide (I I,4 p. Ioo de l'amidon digestible ont été dégradés dans l'intestin contre 7,2 p. I00). Fin revanche, la proportion des matières azotées digérées dans l'intestin (p. Ioo des matières azotées digestibles) a été la même quelle que soit la ration ( 87 p. Ioo).

L'acidité volatile totale du jus de rumen (valeur moyenne de 6 prélèrements effectués au cours des 8 heures qui suivent le repas) a été peu différente selon le régime (tabl. 4) et les quantités de matière organique digérées dans le rumen ont été également sensiblement les mêmes ( 700 et $\mathbf{I} 720 \mathrm{~g}$ en $\mathbf{r} 2$ heures). Ces résultats indiquent que 


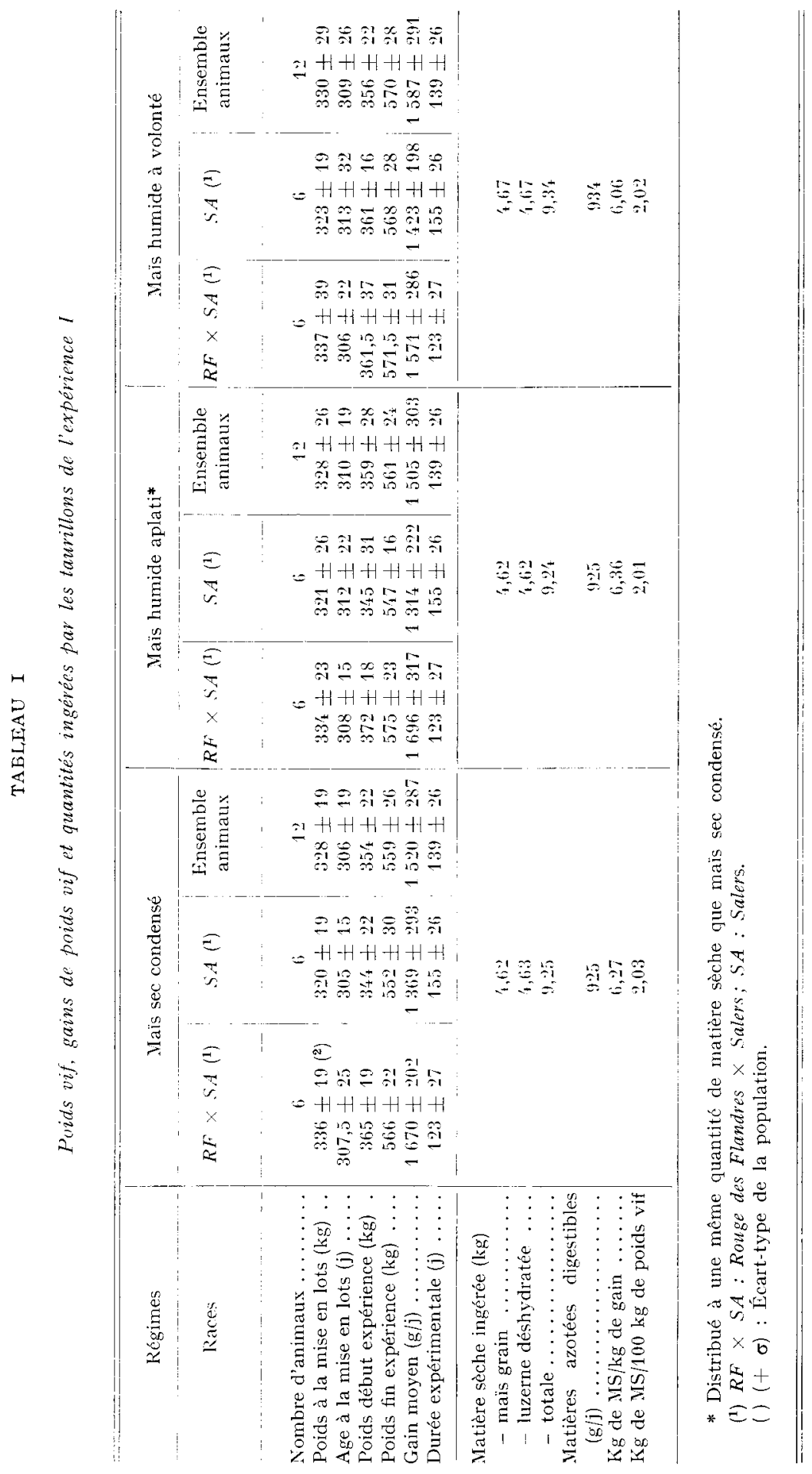


MAIIS GRAIN HUMIDE POUR LES TAURILLONS

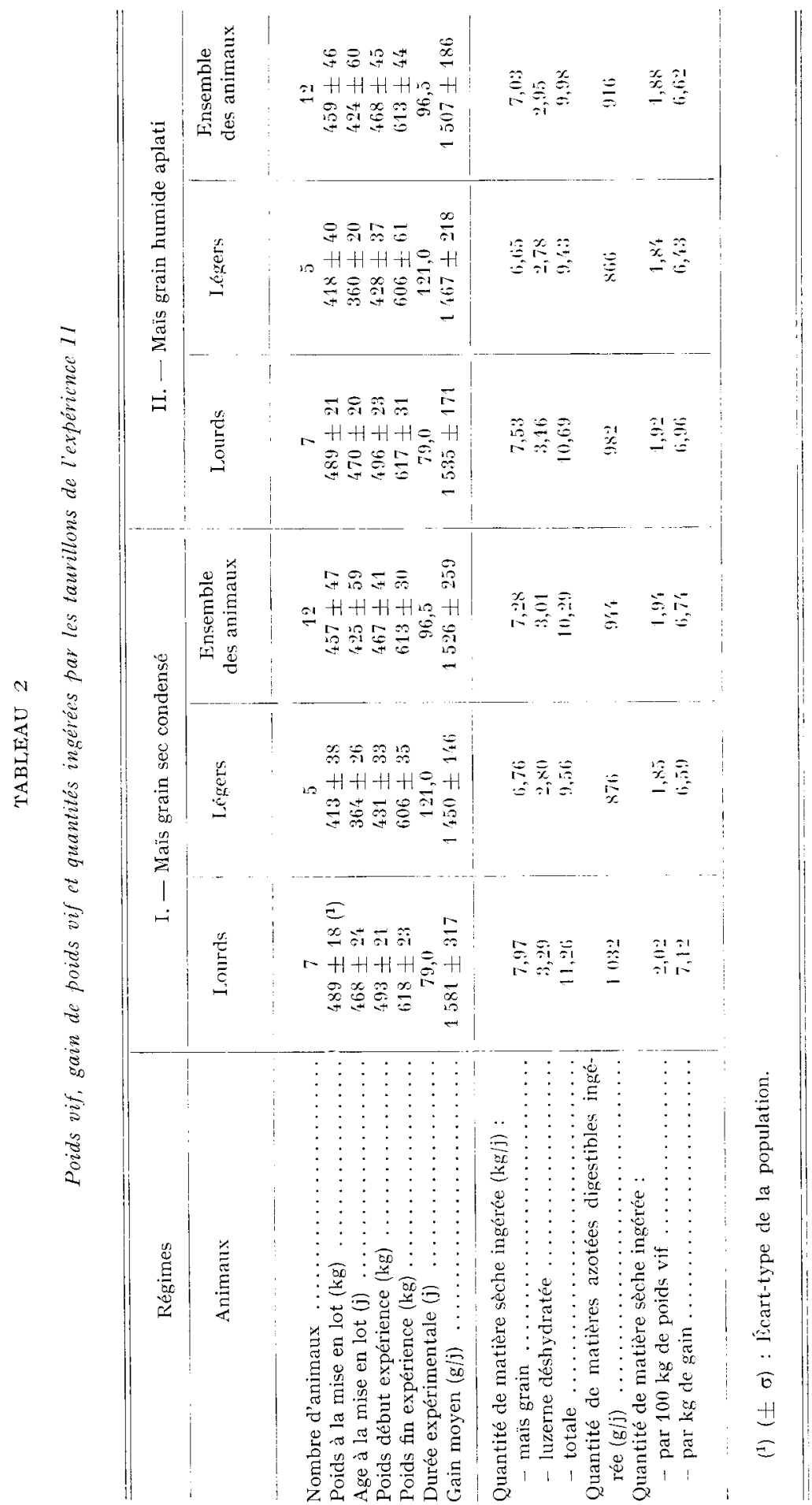


TABLEAU 3

Ltilisation digrstive des régimes (p. 100 de l'ingéré)

\begin{tabular}{|c|c|c|c|c|c|c|c|c|}
\hline & \multicolumn{4}{|c|}{ Dans l'ensemble du tube digestif (1) } & \multicolumn{4}{|c|}{ Dans les réservoirs gastriques (1) } \\
\hline & $\begin{array}{l}\text { Matière } \\
\text { sèche }\end{array}$ & $\begin{array}{l}\text { Matière } \\
\text { organique }\end{array}$ & $\begin{array}{c}\text { Matières } \\
\text { azotćes }\end{array}$ & Amiclon & $\begin{array}{l}\text { Matière } \\
\text { sèche }\end{array}$ & $\begin{array}{l}\text { Matière } \\
\text { organigue }\end{array}$ & $\begin{array}{c}\text { Matières } \\
\text { azotées }\end{array}$ & Amiclon \\
\hline Mais sec condensé & 169,8 & $z_{1, i}$ & $1 ; 2, x$ & ! & $57,: 3$ & (6i), & $\therefore, i$ & 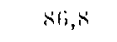 \\
\hline $\begin{array}{l}\text { Mais humide } \\
\text { à volonté }\end{array}$ & 65,7 & 67,7 & 38,5 & 96 & $\pi i, 9$ & $62,2,6$ & 7,5 & $\therefore i, 7$ \\
\hline
\end{tabular}

(1) Ces données ont été obtenues ave des animanx différents.

la quantité journalière totale d'acides gras volatils produits dans le rumen a été très voisine pour les deux régimes. Iin revanche, les quantités respectives des différents acides gras composant le mélange sont différentes. İ̀n effet, le régime à base de maïs humide a donné naissance à une proportion plus élevée d'acide butyrique et plus faible d'acide acétique. Seule, la teneur moyenne en acide propionique a été identique quel que soit le mode de conservation du mais (tabl. 4).

\section{TABLEAU 4}

Composition molaive et concentration (mM-1) du mélange d'acides gras volatils du jus de rumen

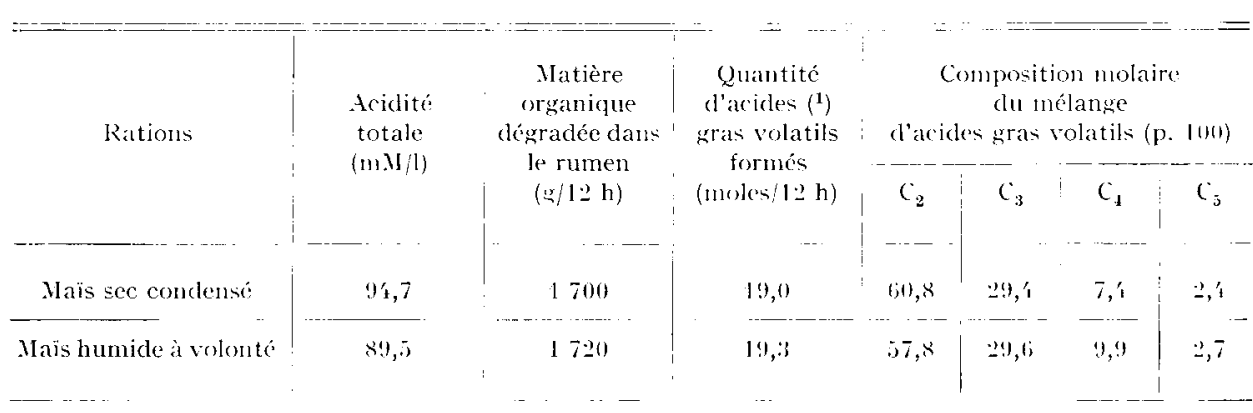

(1) I)'après l.exi: $(1969)$.

Les fermentations postprandiales dans le rumen ont été très régulières avec le régime à base de maïs sec (fig. I a). En revanche, elles ont évolué de façon très différente lorsque les animaux ont reçu le maîs humide : la teneur en acide propionique a augmenté immédiatement après le repas puis a diminué ensuite régulièrement; 
la teneur en acide butyrique a eu une évolution inverse, l'augmentation observée (fig. I a) pouvant être reliée à l'apparition de quantités importantes de sucres solubles dans le jus de rumen (fig. I $b$ ).
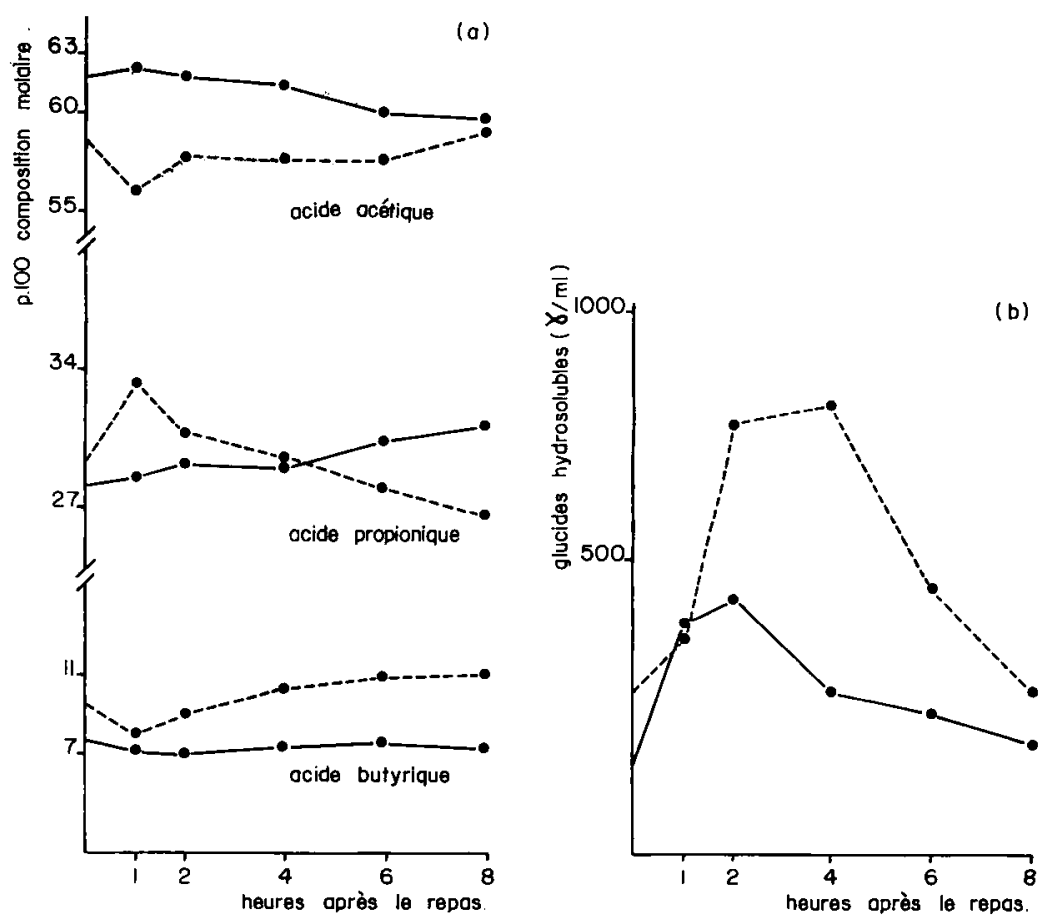

Fic. 1. - Erolution postprandiale de la composition molaire du mélange d'acides gras volatils (a)

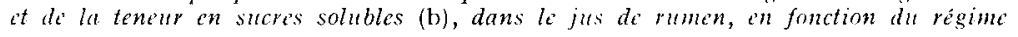

-....- mais sec

\section{Résultats d'abattage}

1)ans les deux essais, le régime n'a pas eu d'influence significative sur le poids de carcasse, le rendement en carcasse (poids de carcasse chaude rapporté au poids vif vide) et la composition de ces carcasses ainsi que sur la proportion de gras du $5^{\circ}$ quartier dans le corps vide (tabl. 5).

\section{DISCUSSION}

Le traitement à l'acide propionique a permis, comme l'avaient observé PERIZ, Michalfasaj et PREston ( 1969 ) sur le sorghograin, de conserver le maïs humide durant 9 1110is, sans diminution de sa teneur en amidon, avec une proportion de pertes relativement faible lors de la $\mathrm{I}^{\mathrm{re}}$ expérience et très faible lors de la seconde.

I,e maïs traité a été bien accepté par les animaux, mais il a été ingéré toutefois 
en quantités légèrement plus faibles que le maïs sec dans l'expérience $n^{0} 2$. Forsytri, Mowat et STONis (I972) ont observé également que des bouvillons recevant du maïs humide broyé, traité à l'acide propionique, ingéraient 3,2 p. Ioo de matière sèche en moins que leurs homologues recevant le maïs sec broyé. Cette légère diminution de l'appétit peut être due à l'apport d'acide propionique alimentaire qui a été relativement important (de 6 à 8,5 p. Ioo de la quantité totale formée dans le rumen) et qui est rraisemblablement responsable de l'augmentation de la teneur en acide propionique du jus de rumen, observée une heure après le repas (fig. I $a$ ). BAUMGARD' (r970) a en effet suggété que la quantité d'acide propionique absorbée, au niveau du tube cligestif, pouvait être un facteur de contrôle de l'appétit du ruminant. Dowdex et Jacobson (I960), BaIre et Meyer (I969) ont également montré qu'une élévation du taux d'acide propionique sanguin entraînait, chez le ruminant, une diminution significative de l'ingestion.

Le traitement du maîs grain à 1'acide propionique a légèrement amélioré 1'efficacité alimentaire de la ration comme le montre la diminution des quantités de matière sèche ingérées par $\mathrm{kg}$ de gain. Ces résultats sont en accord avec ceux de ForsyTH, Mowat et STONL ( I 972). Cependant, ces auteurs ont en outre constaté une augmentation significative de l'état d'engraissement des carcasses que nous n'avons pas observée dans nos deux expériences. Une amélioration (7 p. Ioo) de l'efficacité alimentaire, due à une diminution des quantités ingérées pour un même gain de poids vif, a été également constatée par l'Institut Technique des Céréales et des Fourrages au cours de $f$ essais successifs en $1972-73-74$ et 75 .

Sur le plan énergétique, en estimant les quantités d'énergie métabolisable ingérées par $\mathrm{kg}$ de gain (compte tenu de l'énergie métabolisable apportée par l'acide propionique utilisé lors du traitement : $0,5 \mathrm{Mcal}$ ), on constate que 1'efficacité alimentaire a été améliorée de $7,5 \mathrm{p}$. Ioo pour les animaux recerant le maïs humide à volonté. Cette amélioration peut s'expliquer par l'orientation des fermentations du rumen favorable à une meilleure utilisation de l'énergie (augmentation de la production d'acide butyrique, diminution de la production d'acide acétique) que nous avons constatée chez les animaux recevant le maïs humide.

Mais l'effet bénéfique érentuel sur l'engraissement des animaux a pu être contrebalancé par une diminution de la production de glucose puisque la digestion intestinale de l'amidon a été moins importante chez les animaux recevant le maïs humide

La diminution de digestibilité des matières azotées de la ration de mais humide n'a eu aucune incidence sur le gain de poids vif des animaux, puisque les quantités de matières azotées digestibles ont été très supérieures aux recommandations propo-

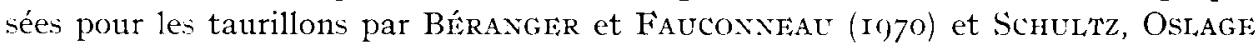
et DAFÁRE (I974).

Ein conclusion, la conservation du maïs grain humide à l'acicle propionique est une technique qui apparait simple et efficace. L'acide propionique diminue peu l'acceptabilité dı grain et entraîne parfois une légère anélioration de l'efficacité alimentaire, sans modifier la composition des carcasses. Dans ces conditions, seul le coût du traitement, par rapport au cô̂t du séchage, peut décider de l'opportunité d'utiliser cette céréale traitée dans l'alimentation du ruminant. 


\section{REMERCIEMENTS}

Cette étude a été réalisée grâce à l'aide dle la Société l'rogil à qui nous exprimons nos remerciements. Nous tenons également à remercier la Station de Recherches sur la Physiologie de la Reproduction qui nous a permis de réaliser la $2^{\mathrm{C}}$ expérience dans son domaine de Bressonvilliers. et tout particulièrement $\mathrm{J}$. PeLot responsable de ce domaine.

\section{SUMMARY}

\section{HIGH MOISTURE MAIZE TREATED WITH PROPIONIC ACID FOR FATTENING YOUNG BULLS}

Two fattening trials were carried out on young bulls to study the effect of processing moist maize with propionic acid on its nutritive value. In each case, the experimental group receiving treated and rolled moist maize was compared with a control group receiving dry, ground and pelleted maize. The maize represented $50 \mathrm{p}$. I00 of the dictary dry matter during experiment I and 70 p. Ioo during experiment 2, the remainder of the diet being composed of dry, ground and pelleted lucerne.

Sixty young bulls were fattened during these 2 experiments lasting 138 and 96 days, respectively. The first experiment included 3 groups of animals ; 2 of these groups received equal amounts of either moist or dry malize ad libitum. The second experiment included 2 groups receiving either dry maize or moist, rolled maize.

Propionic acid allowed to preserve the moist maize (68 and 64,5 p. roo dry matter) for a period of 9 months with only very small losses.

During these two experiments, we did not notice any significant influence of the treatment on live weight gain, weight and carcass composition, but a slight decrease in feed intake and consequently a slight improvement of feed efficiency ( $\mathrm{s} .8$ and $3.3 \mathrm{p} .100$ ).

The treatment with propionic acid brought about a substantial decrease in the chigestibility of the main components of the diet. The amount of volatile fatty acids developed in the rumen was identical, whereas the composition of the rolatile fatty acid mixture was favourably changed, leading to a better energy utilization (increase of butyric acid and decrease of acetic acid) when the animals were fecl moist maize.

Although these results do not show any significant differences, they indicate however in each experiment a slight improvement in the nutritive value of moist matize when preserved with propionic acid.

\section{RÉFÉRENCES BIBLIOGRAPHIQUES}

Bane C. A., Masek J., 1969. Depression of feed intake of goats by metabolites injected durimg neals. Am. J. Physiol., 27, $1830-1836$.

Batagarot B. R., r970. Voluntary feed intalie by runinants : morels and practical applications. Cornell Nutr. Conf., $85-92$.

BeEsos W. M., PERry T. W., I $95 \%$. The comparative feeding value of high moisture corn and low moisture corn with different feed arlditives for fattening beef cattle. J. Anim. Sci., 17, 368-373.

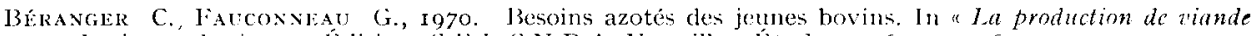
par les jeunes botins" ". liditions S.İ.I.-C.N.R.A. Versailles. Étude no 46, I95-196.

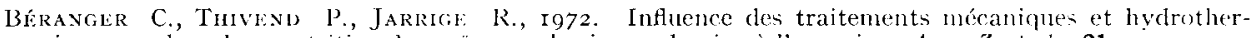
miques sur la valeur nutritive du naiss pour les jeunes bovins à l'engrais. Ann. Zootech., 21, I75-I 9o.

3. P'Chemicals, ig68. Feeding trials. Popcorn treated moist barley r $965-68,9 \mathrm{p}$.

I)Ais C. L., 1967. Acetate production in the rumen of the cows fed either control or low fiber high grain diets. J. Dairy sici., 50, I62 I-1 625 .

I) WDLN D. R., JACOBsox R., I960. Inhibition of appetite in dairy cows by certain intermerliate meta-

bolites. Naturc, 188, I48-I 49 .

Jumont B. I., I956. La découpe du pan traité. F.E.Z. Commission borine, Madrid. 


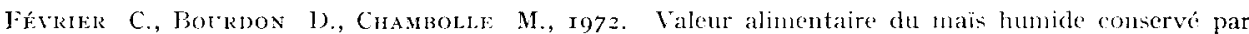
l'acide propionique pour la liruie et le Porcelet, et du mais misilé pour le Porc on croissance-finition. Journées Recherche Porcine en France, 143-147, I.X.R.A.I.T.P., éd.

Fontanot J.-P., l[opkins H. A, 1965. Effect of physical form of different parts of lanb fattening rations on feedlot performance and digestibility. J. Anim. Sci., 24, 6z-68.

Forsim J. (i., Iowat 1). ․, Stone J. l3., 1972. leeding value for beef and dairy cattle of high moisture corn preserved with propionic acid. Can. J. Anim. Sci., 52, 73-79.

GEAY Y., BÉRANGIER C., I969. Estimation de la composition de la carcasse de jeunes bovins ì partit: de la composition d'un morceau monocostal au niveau de la ir e cotc. Ann. Zootech, 18, 65-77.

HAxke II. F., Jornax R. M., r963. Comparison of lambs foed shelled corn and whole or pelleted barley of different bushel weights. J. Anim. Sci, 22, rog7-1099.

I.T.C.F-1.N.A., I972. Vaux-sur-Aure $n^{0}: 0$. Vtilisation du mäs gratin pour la production de jeunes bovins.

I.'T.C.F.-I.X.A., I973. Iaux-sur-Aurc n'30. Utilisation du mais grain pour la production de jeunes bovins.

I.T.C.I., r974. Boigneville $n^{\circ}$ 50. Utilisation du mais sme differentes forme: pour la production de jeunes bovins.

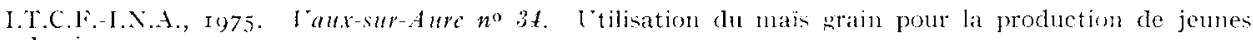
bovins.

Joxes (i. M., IONEFer L., Jillot J. I., I970. Teeding value for dairy cattle and pixs of high moisture corn preserved with propionic acid. Can.J. Anim. Sci., 50, 483-489).

Lelong C., Moni. J., I973. Utilisation du maïs humide traité a l'acide propionique par les bovins et les pores. Ann. Technol. Agric., 22, $64 \mathrm{I}-646$.

MacCafiree J. I), Merriti. W. G., igh6. The feeding value of high noisture corn for dairy cows. Corncll Nutr. (onf., 77-85.

Mackines D. I), KigGs J. K., 1967. Moist workhum grains for dinishing attle. J. Anm. Sci., 26, 925 (abstr.).

PÉREZ R., MICUliexAJ, l'REstox T. R., 1969. Relative value for broilers of sorghum grain with high moisture content ensiled or preserved with propionic acid. Re?. Cubana ricnc. Agric., 3, I85-I88.

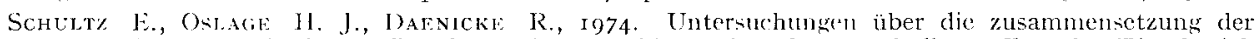
korpersubstan\% sowic den stoff-und onervicansaty bie wahsenden mastbullen. Fortschr. Tierphysiol. Tiercrnähr., 4.

Thérnez M., LF ] J. Jo, Móxat G., I973. Comparaison de quatre inćthedes de conservation du maiss grain. Influence sur la valeur alimentaire pour l'engraisement de l'Asneau. Ann. Zootech., 22, $443-440$.

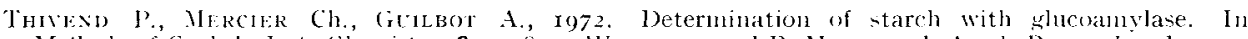

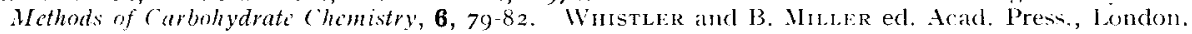

Vissac 13., I959. Rapport sur des recherches lirançaises intéressint le testage des taureaux sur les aptitudes a la production de viande de leurs descendants. C. K. des Journés d' E.tudes de la IF.E.Z., $n^{\circ} 700 / 59$, Be]grade.

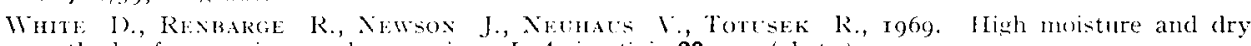
methods of processing sorghum grain. J. Anim. tici, 29, I75 (abstr.).

Zogo C. A., Bkowx R. li., Harshbarger K. l., Kendat. K. A., I96r. Nutritive value of high molisture conn when fed with variouls silages to lactating dairy cows. J. Dairy Sici., 44, 4r3-490. 